\title{
PREVALÊNCIA DO ECZEMA E FATORES ASSOCIADOS EM UMA CIDADE DO NORDESTE DO BRASIL
}

\author{
ECZEMA PREVALENCE AND ASSOCIATED FACTORS IN A CITY IN BRAZIL'S \\ NORTHEAST
}

\section{PREVALENCIA DEL ECCEMA Y FACTORES ASOSIADOS EN UNA CIUDAD DEL NORESTE DE BRASIL}

\begin{abstract}
Diego Augusto Medeiros Santos ${ }^{1}$, Kelvyn Melo Vital ${ }^{2}$, Ana Carolina Neves Costa e Silva ${ }^{3}$, Emily Anara Oliveira Nascimento ${ }^{4}$, Auxiliadora Damianne Pereira Vieira Costa ${ }^{5}$, Mércia Lamenha Medeiros ${ }^{6}$
\end{abstract}

\begin{abstract}
RESUMO
Objetivos: identificar a prevalência e analisar os fatores associados ao eczema entre adolescentes de 13 e 14 anos em Maceió, uma capital do Nordeste brasileiro. Método: trata-se de um estudo transversal, participante do Internacional Study of Asthma and Allergies in Childhood (ISAAC), examinando adolescentes de escolas públicas e privadas, com coletas realizadas em dois momentos, 2002 e 2012. A análise estatística descritiva foi realizada por meio de frequências simples, média e desvio-padrão. Para o estudo dos fatores associados, utilizou-se análise bivariada por meio de OR - razão de chance e quiquadrado. Resultados: a prevalência do eczema foi de 7,5\% (2002) e de 4\% (2012). Na análise bivariada entre os portadores de eczema, foram identificados os seguintes fatores associados: ter ambos os pais com eczema; tipo de piso; uso de travesseiro; ter irmão mais novo; frequentar creches e hábitos alimentares como o consumo de peixes, frutas e aleitamento materno até quatro meses de vida. Conclusão: este é o primeiro estudo, em Maceió, do qual se tem nota, com o objetivo de avaliar a prevalência em dois momentos, bem como tentar identificar fatores associados ao eczema entre adolescentes. Os dados encontrados podem auxiliar os profissionais da saúde, portadores e familiares quanto a uma melhor compreensão da enfermidade e dos fatores associados.
\end{abstract}

Palavras-chave: Eczema; Hereditariedade; Fatores de Risco.

\section{ABSTRACT}

Objective: to identify prevalence and analyze factors associated with eczema among 13and 14-year-old adolescents in Maceió, a capital of northeastern Brazil. Method: this is a cross-sectional study, participating in the International Study of Asthma and Allergies in

\footnotetext{
${ }^{1}$ Residente. Universidade de São Paulo. Ribeirão Preto (SP), Brasil.

2,3,4 Médicos. Universidade Federal de Alagoas. Maceió ( $A L)$, Brasil.

5,6 Doutores. Universidade Federal de Alagoas. Maceió (AL), Brasil.
} 
Childhood (ISAAC), examining adolescents from public and private schools, with collections performed in two moments, 2002 and 2012. Descriptive statistical analysis was performed using simple frequencies, mean and standard deviation. For the study of associated factors, bivariate analysis was used by OR - odds ratio and chi-square. Results: the prevalence of eczema was $7.5 \%$ (2002) and 4\% (2012). In the bivariate analysis among eczema patients, the following associated factors were identified: having both parents with eczema; floor type; pillow use; have younger brother; attend daycare centers and eating habits such as eating fish, fruits and breastfeeding up to four months of life. Conclusion: this is the first study, in Maceió, of which it is noted, aiming to evaluate the prevalence in two moments, as well as to try to identify factors associated with eczema among adolescents. The data found may help health professionals, patients and families to better understand the disease and associated factors.

Keyword: Eczema; Heredity; Risk Factors.

\section{RESUMEN}

Objetivo: identificar la prevalencia y analizar los factores asociados con el eccema entre los adolescentes de 13 y 14 años en Maceió, una capital del Noreste de Brasil. Método: es un estudio transversal, participante del Internacional Study of Asthmaand Allergies in Childhood (ISAAC), examinando adolescentes de escuelas públicas y privadas, con recolección realizadas en dos momentos, 2002 y 2012. El análisis estadístico descriptivo a través de frecuencias simples, mediana y desviación estándar. Para el estudio de los factores asociados, se utilizó el análisis bivariado por OR-chance ratio y Chi-cuadrado. Resultados: la prevalencia de eccema fue del 7,5\% (2002) y del 4\% (2012). En el análisis bivariado entre pacientes con eccema, se identificaron los siguientes factores asociados: tener a ambos padres con eccema; tipo de piso; uso de almohadas; tener hermanos menores; frecuentar a guarderías y hábitos alimenticios como el consumo de pescados, frutas y lactancia materna hasta cuatro meses de vida. Conclusión: este es el primer estudio, en Maceió, que hemos observado, con el objetivo de evaluar la prevalencia en dos momentos, así como tratar de identificar los factores asociados con el eccema entre los adolescentes. Los datos encontrados pueden ayudar a los profesionales de la salud, portadores y familiares a comprender mejor la enfermedad y los factores asociados.

Palabras-clave: Eccema; Herencia; Factores de Riesgo.

\section{INTRODUÇÃO}

O Eczema Atópico (EA) é uma doença inflamatória crônica da pele e é uma das patologias mais prevalentes, com variabilidade geográfica mundial entre $1 \%$ e $20 \% .{ }^{1}$ Caracterizado pelo sintoma de prurido intenso acompanhado de lesões eczematosas, precisa de atenção por ter grande impacto nos aspectos sociais e psicológicos. ${ }^{2}$

A prevalência e os fatores de risco para o eczema foram compreendidos graças ao International Study of Asthma and Allergies in Childhood (ISAAC), que tinha como objetivo estudar asma e doenças alérgicas a partir de questionários padronizados. ${ }^{1,3} \mathrm{O}$ estudo foi desenvolvido em três fases: avaliar a prevalência e gravidade da asma e doenças atópicas; identificar possíveis fatores de risco relacionados aos achados da fase I; repetição da fase I após um período de cinco 
anos para avaliar as tendências temporais da prevalência dessas doenças. ${ }^{3} \mathrm{O}$ ISAAC permitiu que houvesse uma comparação entre 153 centros de 56 países nos quais a prevalência do eczema foi sempre maior para crianças menores. ${ }^{4}$

Neste estudo multicêntrico (ISAAC), buscava-se compreender como as doenças atópicas se comportavam nos diversos países e continentes. Atopia é uma predisposição hereditária do sistema imune a privilegiar reações de hipersensibilidade do tipo I, ou seja, aquelas que são mediadas por IgE após estimulação de mastócitos proporcionada por uma estimulação Th2. Essa reação imune ocorre em resposta a antígenos - ou alérgenos - que são comuns ao ambiente domiciliar e até mesmo ao ambiente externo com o qual o indivíduo tem contato. ${ }^{4-5}$ Inicialmente, o termo atopia relacionava-se apenas com asma e rinite, mas, dez anos depois, em 1933, os responsáveis pelo termo (Coca e Cooke) classificaram também a dermatite atópica como atopia pela semelhança de padrão. ${ }^{6}$

Os alérgenos são substâncias químicas que contêm um caráter proteico, seja por sua própria constituição ou por associação a proteínas glicosiladas que são solúveis nos fluidos corporais. Dessa maneira, os alérgenos mantêm-se protegidos de desnaturação e de degradação, o que possibilita que sejam absorvidos intactos. ${ }^{6}$

Os estudos sobre os fatores genéticos, ambientais e dietéticos são relevantes, tendo em vista a participação destes na determinação de doenças atópicas e na forma como elas se manifestam em diversos indivíduos. ${ }^{6} \mathrm{O}$ eczema possui caráter genético multifatorial, o que torna o estudo mais complexo. ${ }^{7}$ Os fatores ambientais que são estudados neste trabalho incluem a exposição a mofo e a ambiente interno. Aspectos ligados ao aleitamento e dieta podem estar relacionados às variações.

As doenças atópicas, em geral, tiveram um aumento na prevalência, uma vez que os hábitos de vida mudaram, tornando-se mais propícios para seu desencadeamento. As formas de moradia, o uso de cortinas e almofadas, novos hábitos de alimentação e grandes preocupações com higiene podem ter contribuído com o aumento dos índices dessas atopias ou, até mesmo, aumentado a sua morbidade. ${ }^{8}$

A participação dos centros de pesquisas sob o protocolo ISAAC contribuiu para a maior representatividade dos dados, em níveis regional e nacional, favorecendo as comparações. Este estudo teve como objetivo identificar a 
prevalência do eczema e os possíveis fatores associados ao eczema entre adolescentes de 13 e 14 anos moradores de uma cidade do Nordeste brasileiro.

\section{MÉTODO}

\section{Desenho e Local do Estudo}

Estudo transversal, realizado na cidade de Maceió( $A L)$, por meio da aplicação de questionários escritos, traduzidos e validados do ISAAC.

\section{Seleção dos Participantes}

A amostra estabelecida pelo ISAAC era de 3000 crianças (de seis e sete anos) e de 3500 adolescentes (de 13 e 14 anos). Com o objetivo de localizar os investigados, constituíram-se como sistema de referência os sete distritos em que se divide a cidade de Maceió( $A L)$, fornecidos pela secretária municipal de Educação, respeitando-se a proporcionalidade entre o número de escolas públicas e privadas, para proceder ao sorteio das escolas e das turmas. Foram critérios de inclusão crianças com seis e sete anos e adolescentes de 13 e 14 anos, alfabetizados e que tivessem condições cognitivas de responder às questões. Foram excluídos os portadores de doenças cardiorrespiratórias e dermatológicas graves, questionários incorretos ou incompletos.

\section{Instrumento e Definição das Variáveis}

Os participantes foram informados sobre a pesquisa e assinaram o Termo de Consentimento Livre e Esclarecido (TCLE). Os questionários iniciais continham questões sobre sintomas e gravidade para asma, rinite e eczema. O critério diagnóstico para eczema (dermatite atópica) era a resposta "sim" à presença de "rash pruriginoso que aparece e desaparece nos últimos 12 meses".

Foram identificados os portadores do eczema e feito o sorteio do grupocontrole da mesma série e escola. Nessa segunda etapa, foram enviados, aos responsáveis, o TCLE e um questionário composto de 33 questões referentes às condições de nascimento, imunização, alimentação, vacinação, fatores socioeconômicos, ambientais, animais domésticos, irmãos, tabagismo e antecedentes familiares.

\section{Análise Estatística}


Os dados foram transcritos para o banco de dados EPIINFO, versão 3.5.4, por meio de dupla entrada. As frequências de respostas a cada questão foram obtidas em relação ao número total de questionários válidos. As associações entre gênero, tipo de escola e nível socioeconômico (estratos de renda familiar e escolaridade materna) foram analisadas utilizando-se o teste do qui-quadrado $\left(X^{2}\right)$. O nível de significância estabelecido foi de $5 \%(p<0,05)$. A estatística conta com uma parte descritiva por meio de frequências simples, média e desvio-padrão, e uma parte analítica usando análise bivariada por meio de OR-Razão de chance, qui-quadrado, cujos resultados nortearam a seleção das variáveis que participaram da análise multivariada por meio da Regressão Logística Dicotômica.

$\mathrm{Na}$ análise multivariada, utilizaram-se as variáveis que tiveram um $p<0,20 \mathrm{e}$ mais aquelas que eram importantes na determinação dos efeitos ou que pudessem estar relacionadas com interação ou confundimento.

\section{Considerações Éticas}

Os questionários incorretamente preenchidos foram excluídos do estudo. A pesquisa foi aprovada pelo comitê de ética em pesquisa da Universidade Federal de Alagoas sob o protocolo 005247/2010-43.

\section{RESULTADOS}

\section{Prevalência do Eczema}

Na primeira etapa do ISAAC (2002), obteve-se uma amostra válida com $66,4 \%$ das crianças (de seis e sete anos) e entre 92\% dos adolescentes (de 13 e 14 anos). Encontrou-se a prevalência de EA, que foi de 10,5\% nas crianças e de $7,5 \%$ nos adolescentes, descobrindo-se associação com o gênero feminino, com $\mathrm{p}<0,00$ de significância estatística (Figura 1).

O estudo epidemiológico ISAAC foi retomado em 2012, com 93,4\% questionários considerados válidos, em uma população de 3500 adolescentes. A prevalência do eczema entre os adolescentes foi de 4\% (130), que responderam "sim" à presença de "rash pruriginoso que aparece e desaparece nos últimos 12 meses". 
Figura 1. Prevalência de Eczema de Acordo com o Gênero entre Escolares

e Adolescentes. $(P<0,00)$

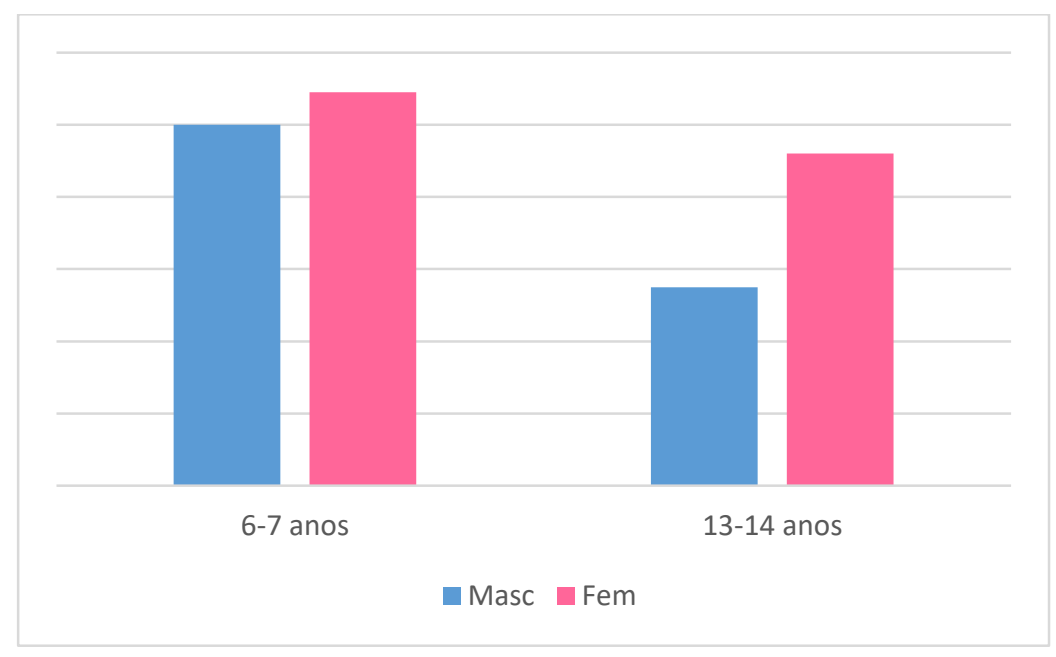

\section{Fatores Associados ao Eczema}

Neste mesmo estudo, dos 450 estudantes avaliados quanto à presença de fatores de risco, 74 apresentavam eczema e 376 foram os caso-controle. Ter amamentado por, no mínimo, dois a quatro meses, frequentado o jardim de infância antes dos dois anos e ter piso liso (sem carpetes) foram considerados fatores de proteção, reduzindo, pela metade, a chance de ter eczema na amostra estudada. Por outro lado, o não consumo de frutas frescas e peixes, ter mãe e pai (ambos) com eczema, não usar travesseiro com capa protetora e ser o mais velho de, pelo menos, quatro irmãos mais novos estiveram associados a chances de duas a quatro vezes em ter a afecção (Tabela 1). Houve uma tendência a efeito protetor do gênero masculino. 
Tabela 1. Prevalência dos Fatores Associados ao Eczema entre Adolescentes.

\begin{tabular}{|c|c|c|c|c|}
\hline \multirow[t]{2}{*}{ Variáveis } & \multicolumn{2}{|c|}{ ECZEMA (450) } & \multirow[t]{2}{*}{ OR (IC 95\%) } & \multirow[t]{2}{*}{$\boldsymbol{P}$} \\
\hline & $\begin{array}{c}\text { Sim } \\
(74) \\
\mathbf{N}(\%) \\
\end{array}$ & $\begin{array}{c}\text { Não } \\
(376) \\
N(\%) \\
\end{array}$ & & \\
\hline \multicolumn{5}{|l|}{ Demográficos } \\
\hline Sexo masculino & $19(25,7)$ & $141(37,5)$ & $0,58(0,33-1,01)$ & 0,05 \\
\hline \multicolumn{5}{|l|}{ Hábitos alimentares } \\
\hline $\begin{array}{l}\text { Aleitamento Materno } 2 \text { a } 4 \\
\text { meses }\end{array}$ & $11(14,8)$ & $104(27,6)$ & $0,45(0,22-0,89)$ & $0,02 *$ \\
\hline Nunca consome peixe & $14(18,9)$ & $33(8,7)$ & $2,39(1,18-4,81)$ & $0,01 *$ \\
\hline Nunca consome suco de frutas & $7(9,4)$ & $39(10,4)$ & $3,46(1,43-8,39)$ & $0,00 *$ \\
\hline \multicolumn{5}{|l|}{ Familiares } \\
\hline Ambos os pais com asma & $10(13,5)$ & $63(16,7)$ & $0,78(0,35-1,66)$ & 0,49 \\
\hline Ambos os pais com eczema & $8(10,8)$ & $16(4,2)$ & $2,73(1,02-7,11)$ & $0,02 *$ \\
\hline \multicolumn{5}{|l|}{ Ambientais } \\
\hline Reduziu tabagismo em casa & $5(6,7)$ & $9(2,4)$ & $2,52(0,80-7,96)$ & 0,10 \\
\hline Não usa travesseiro no presente & $8(10,8)$ & $14(3,7)$ & $3,13(1,15-8,37)$ & $0,02 *$ \\
\hline Piso liso $1^{\circ}$ ano/presente & $29(39,2)$ & $193(51,3)$ & $0,55(0,32-0,96)$ & $0,04 *$ \\
\hline Removeu animais em casa & $9(12,2)$ & $22(5,8)$ & $2,17(0,94-5,02)$ & 0,06 \\
\hline Presença de mofo na casa & $15(20,3)$ & $55(14,6)$ & $1,48(0,75-2,91)$ & 0,22 \\
\hline Jardim de infância $<2$ anos & $5(6,7)$ & $71(18,9)$ & $0,44(0,20-0,99)$ & $0,04 *$ \\
\hline Mais de 4 irmãos mais novos & $9(12,2)$ & $12(3,2)$ & $4,48(1,75-11,48)$ & $0,00 *$ \\
\hline \multicolumn{5}{|l|}{ Socioeconômicos } \\
\hline Renda $<5$ SM & $63(85,1)$ & $304(80,8)$ & $1,36(0,65-2,88)$ & 0,38 \\
\hline Escolaridade Materna $<8$ anos & $28(37,8)$ & $136(36,2)$ & $1,04(0,60-1,82)$ & 0,87 \\
\hline
\end{tabular}

\section{DISCUSSÃO}

As prevalências do eczema entre os adolescentes declinaram em dez anos, dados coerentes com alguns estudos, que também encontraram essa redução, cujos autores atribuíram aos fatores ambientais, que são fundamentais na expressão do eczema, por serem improváveis mudanças genéticas em um período tão curto de tempo (de cinco a dez anos). Observou-se prevalência mais elevada entre as crianças, quando analisadas em 2002, e, na literatura, verificou-se que, entre $30 \%$ a $50 \%$ dos pacientes EA, a doença melhora até chegar a adolescência. ${ }^{2}$

Neste estudo, quando ambos os pais tinham eczema, houve quase três vezes mais chances de adolescentes de terem eczema. A interação de herança genética com alergias já é conhecida, mas ainda é uma dificuldade conhecer os polimorfismos dos vários genes, que interagem com diversos outros fatores, sejam ambientais, pessoais e hábitos de vida. ${ }^{9}$ Existe uma grande quantidade de genes associados, como é o caso do gene da filagrina. ${ }^{10}$ 
O eczema é deflagrado por defeitos na barreira cutânea que facilitam a entrada de alérgenos e substâncias irritantes, causados, em grande parte, por mutações no gene da filagrina, uma das proteínas responsáveis pela integridade cutânea. ${ }^{9,11-12}$ Essas mutações ganharam grande destaque nos estudos das bases genéticas da doença, embora não estejam presentes em todos os casos, portanto, não podem ser consideradas a única responsável pelo desenvolvimento dessa atopia. ${ }^{11-12}$

A amamentação deve ser exclusiva até os seis meses de vida por inúmeros aspectos, como aumentar o laço afetivo entre as mães e seus bebês, proporcionar aumento da proteção contra doenças crônicas, infecciosas e reduzir as doenças imunoalérgicas, diminuindo, assim, a morbimortalidade infantil. ${ }^{13-14}$ O efeito protetor do aleitamento materno exclusivo diz respeito ao desenvolvimento de uma flora intestinal rica em probióticos, dificultando o transporte de antígenos na mucosa, uma vez que essa se torna menos permeável, reduzindo, assim, o desenvolvimento de alergias. ${ }^{15}$

Os achados deste estudo revelaram associação no uso do aleitamento materno, entre dois a quatro meses de vida, inclusive, com significância estatística ( $\mathrm{p} 0,02)$, dados coerentes com os de estudos que evidenciaram essa variável como fator de proteção no desenvolvimento de atopias,${ }^{16}$ sendo que a exclusividade até os quatro meses de vida destacou-se como tempo mínimo para a diminuição do risco. ${ }^{17}$ Consolidam-se esses achados pela literatura, que descreveu que o uso de fórmulas lácteas infantis (mesmo isenta de leite de vaca e proteínas de soja), se comparadas com o leite materno, ocasionou maior incidência de dermatite atópica. ${ }^{18}$

Investigaram a relação da amamentação e a sensibilização a aeroalérgenos, demonstrando que o genótipo de diferenciação (CD14) define o aleitamento materno como proteção ou risco. ${ }^{20}$ De fato, muitos defendem que o aleitamento seja capaz de prevenir alergias e sustentam a teoria dos benefícios do leite materno nesse aspecto, ${ }^{23}$ no entanto, ainda há muitas controvérsias, provavelmente pelos inúmeros aspectos que influenciam a manutenção de um lactente saudável.

O papel dos fatores dietéticos é controverso, pois estudos de observação mostraram que dietas pobres em antioxidantes (vitaminas $A$ e $C$ ), magnésio, selênio e gorduras poli-insaturadas ômega-3 (óleo de peixe), ou ricas em sódio e poli-insaturadas ômega-6, estão associadas ao maior risco de desenvolver asma. 
A deficiência de vitamina $D$ também pode predispor ao desenvolvimento desta doença. Entretanto, estudos de intervenção com suplementos dietéticos não confirmaram que os fatores dietéticos desempenhem um papel importante. ${ }^{21}$ Nos dados deste estudo quanto à alimentação atual, não consumir frutas ou peixes foi associado e significativo para eczema (p 0,004 e 0,013, respectivamente), aumentando o risco para a doença. Por outro lado, alguns estudos sugeriram que determinadas características da dieta ocidental, como o consumo de alimentos processados, em detrimento de frutas e vegetais com efeitos antioxidantes e diminuição de ingestão de ácidos graxos ómega-3, podem estar associadas ao aumento de incidência das doenças alérgicas e da asma. ${ }^{22}$

Tais resultados foram atribuídos ao rico conteúdo de ácidos graxos poliinsaturados, ${ }^{23-24}$ principalmente o ômega 3, que agem na inibição da resposta Th2 e da síntese de interleucinas 2 e 4, responsáveis pelo desenvolvimento de atopias. $^{24}$ Alguns estudos revelaram benefícios relacionados na dieta do mediterrâneo, na qual há um maior consumo de frutas, peixes e azeite de oliva, promovendo menores índices de atopias. ${ }^{15}$

Nas questões ambientais, a presença de irmãos mais novos apresentou-se como aumentando o risco em significância ( $p$ 0,003). Estudo realizado em Itajubá, MG, demonstrou que havia relação de risco, uma vez que foi encontrada maior prevalência de doenças atópicas em crianças que tinham dois a quatro irmãos em relação aos que só tinham um. ${ }^{25}$

Segundo a hipótese higienista, a presença de famílias numerosas e o convívio de crianças no jardim de infância apresentam-se como proteção para o desenvolvimento de atopias devido à maior possibilidade de infecções adquiridas pelo contato entre as crianças, ${ }^{9,20}$ assim como frequentar jardim de infância apresentou-se como fator de proteção ( $p$,041). Essa hipótese considera que um maior número de infecções da infância diminui o desenvolvimento de alergias já que há uma maior estimulação do padrão Th1, como foi observado em estudos nos quais irmãos mais novos eram menos alérgicos do que os mais velhos, pois tinham infecções mais frequentes. ${ }^{10}$ Estudos de coorte também mostraram relação de proteção para a convivência de crianças em creches nos primeiros anos de vida. $^{2}$

Os ácaros são um importante agente causador de alergias, tendo seus alérgenos uma participação na diminuição da barreira do estrato córneo da pele. ${ }^{24}$ Assim, o uso de travesseiros sintéticos, que acumulam uma maior quantidade de 
ácaros, aumenta o risco de desenvolvimento de dermatites atópicas nos primeiros anos de vida. ${ }^{7,24-25}$ O uso de capas em travesseiros e camas reduz significativamente a exposição aos alérgenos e, consequentemente, a gravidade das lesões. ${ }^{26}$ Identificou-se, neste estudo, que o não uso de travesseiros $(p 0,02)$ e o uso de piso liso $(0,04)$ foram significantes. O fato de preferir piso liso pode proporcionar menor acúmulo desses ácaros, ocasionando um menor risco para o EA.

As características ambientais relacionadas ao quarto da criança alérgica e a seu domicílio são associadas ao desenvolvimento de doenças atópicas. Segundo o Guia de Avaliação Ambiental do Alérgico, criado pelo Centro de Orientação em Rinite Alérgica do Hospital das Clínicas da Faculdade de Medicina da Universidade de São Paulo, existem algumas características que devem ser analisadas a fim de classificar o ambiente domiciliar como adequado ou não para o alérgico. A ausência de carpetes, cortinas, tapetes, almofadas e animais, associada à presença de travesseiros com capa, edredom, móveis indispensáveis e paredes livres de mofo, torna o ambiente considerado adequado. ${ }^{27}$

Em relação a sexo, neste estudo, houve maior prevalência de eczema no sexo masculino, porém, não significante ( $p 0,052)$. Diversos estudos presentes na literatura trazem o sexo masculino como mais prevalente entre as doenças atópicas. ${ }^{20}$ Segundo estudo feito em Criciúma, Santa Catarina, também houve uma maior prevalência nos meninos. ${ }^{28}$

Diante do exposto, observou-se a necessidade de novas pesquisas para que se possam consolidar os fatores relacionados ao EA, a fim de conhecer melhor as doenças atópicas, diminuindo, assim, os danos causados por uma doença crônica. ${ }^{29}$ Além disso, conhecendo melhor os fatores de risco associados, é possível trilhar novos caminhos na prevenção primária e secundária, podendo ser possível uma melhor qualidade de vida. ${ }^{30}$

A prevalência da asma e rinite alérgica, associada com quadro de dermatite atópica nos primeiros anos de vida da criança, demonstrou que existe uma relação de risco entre dermatite atópica, asma e rinite alérgica, principalmente quando encontrada em sua forma clínica mais grave e por meio de medidas de associação de risco confiáveis.

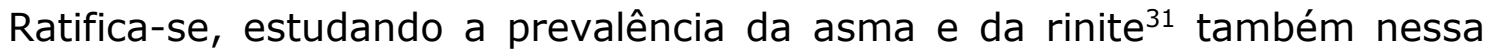
mesma população, publicada anteriormente, e as consequências dessas na qualidade de vida, principalmente na faixa pediátrica e lembrando de considerar 
como possível evolução a marcha atópica, assim como as evidências da relação com fatores associados, a importância das medidas de cuidados na atenção básica, no tratamento e na condução do eczema desde os primeiros anos de vida, a fim de possivelmente prevenir o eczema, a asma e a rinite e garantir a qualidade de vida nos portadores de eczema. ${ }^{3}$ Em estudos multicêntricos internacionais, mesmo validados como este, há sempre o risco da não compreensão do questionário e uma possível confusão com outras dermatites.

\section{CONCLUSÃO}

Este é o primeiro estudo, em Maceió, uma capital do Nordeste do Brasil, do qual se tem nota, com o objetivo de avaliar a prevalência em dois momentos e identificar fatores associados ao eczema entre adolescentes. Os dados encontrados, de redução da prevalência e da associação do eczema com fatores genéticos, alguns ambientais e alimentares, podem auxiliar os portadores, familiares e profissionais da saúde quanto a uma melhor compreensão da enfermidade e dos fatores associados. Destaca-se que o eczema tem relação com a qualidade de vida e precisa-se considerar a marcha atópica como possível evolução.

\section{REFERÊNCIAS}

1. Solé D, Wandalsen GF, Camelo-Nunes IC, Naspitz CK. Prevalence of symptoms of asthma, rhinitis, and atopic eczema among Brazilian children and adolescents identified by the International Study of Asthma and Allergies in Childhood (ISAAC) Phase 3. J Pediatr (Rio J). 2006 Sept/Oct;82(5):341-6. DOI: http://dx.doi.org/10.1590/S0021-75572006000600006

2. Lee SH, Lee SH, Lee SY, Lee B, Lee SH, Park YL. Psychological health status and health related quality of life in adults with atopic dermatitis: a nationwide cross-sectional study in South Korea. Acta Derm Venereol. 2018; 98:89-97. DOI: http://dx.doi.org/10.2340/00015555-2797

3. Solé $D$. International study of asthma and allergies in childhood (ISAAC): o que nos ensinou? J Bras Pneumol. 2005 Mar/Apr; 31(2):93-94. DOI: http://dx.doi.org/10.1590/S180637132005000200001

4. Serpa FS, Zandonade E, Reis JL, Borja TN, Moyses T, Campinhos FL, et al. Prevalence rates of asthma, allergic rhinitis and atopic eczema in schoolchildren in the city of Vitória, Espírito Santo, Brazil. Rev Bras Pesq Saúde. 2014 July/Sept; 16 (3):107-14. DOI: http://dx.doi.org/10.21722/rbps.v16i3.10144

5. Leite RMS, Leite AAC, Costa IMC. Atopic dermatitis: a cutaneous or systemic disease? The search for answers in the history of Dermatology. An Bras Dermatol. 2007 Jan/Feb;82(1):718. DOI: http://dx.doi.org/10.1590/S0365-05962007000100010

6. Boechat JL, França AT. Atopic march. Rev bras alerg imunopatol [Internet]. 2008 [cited 2019 Aug 10]; 31(4):139-45. Available from: http://aaaiasbai.org.br/audiencia_pdf.asp?aid2=195\&nomeArquivo=v31n4a04.pdf\&ano=2008

7. Abbas AK, Lichtman, AH. Alergia e hipersensibilidade. Imunologia Celular e Molecular. 5th ed. Rio de Janeiro: Elsevier; 2005. 
8. Castro APM, Solé D, Rosário Filho NA, Jacob CMA, Rizzo MCFV, Fernandes MFM, et al. Practical guide for management of atopic dermatitis - conjunct opinion of allergologists from the Associação Brasileira de Alergia e Imunopatologia and Sociedade Brasileira de Pediatria. Rev Bras Alerg Imunopatol [Internet]. 2009 Dec [cited 2018 Aug 10]; 29(6):268-82. Available from: http://aaai-asbai.org.br/detalhe_artigo.asp?id=278

9. Nutten S. Dermatitis: global epidemiology and risk factors atopic. Ann Nutr Metab. 2015;66(1):8-16. DOI: http://dx.doi.org/10.1159/000370220

10. Nunes ICC. Hipótese da Higiene: persistir é preciso! Rev Bras Alerg Imunopatol [Internet]. 2011 [cited 2019 Aug 15];34(2):31-2. Available from: http://aaaiasbai.org.br/detalhe_artigo.asp?id=75

11. Barbosa LS. Allergic rhinits and asthma: coexistence and influence household. Investigação. 2010 [cited 2019 Aug 10]; 10(1):13-18. Available from: http://publicacoes.unifran.br/index.php/investigacao/article/view/148

12. Jaramillo-Rodríguez OD, González-Correa CHG. Probiotics in primary prevention of atopic dermatitis in infants at risk of suffering it. Metaanalysis. Biosalud [Internet]. 2013 Jan/June [cited $2019 \quad$ Aug 10]; 12(1):18-28. Available from: http://www.scielo.org.co/pdf/biosa/v12n1/v12n1a03.pdf

13. Cardili RN, Melo JML, Roselino AM, Moreno AS, Castro APM, Arruda LK. Atopic dermatitis and filaggrin: restoring barriers to control the disease. Braz J Allergy Immunol [Internet]. 2013 [cited 2019 Aug 10];1(5):239-42. Available from: http://aaaiasbai.org.br/audiencia_pdf.asp?aid2 $=656 \&$ nomeArquivo $=$ v1n5a02.pdf\&ano $=2013$

14. Lai-Cheong JE, Mcgrath JA. Advances in understanding the genetic basis of inherited single gene skin barrier disorders: new clues to key genes that may be involved in the pathogenesis of atopic dermatitis. An Bras Dermatol. 2006 Nov/Dec; 81(6):567-71. DOI: http://dx.doi.org/10.1590/S0365-05962006000600009

15. Ministério da Saúde (BR), Secretaria de Atenção à Saúde, Departamento de Atenção Básica. Saúde da Criança: Nutrição Infantil: Aleitamento Materno e Alimentação Complementar [Internet]. Brasília: Ministério da Saúde; 2009 [cited 2019 Aug 10]. Available from: http://bvsms.saude.gov.br/bvs/publicacoes/saude_crianca_aleitamento_materno_cab23.pdf

16. Cocco RR, Souza FS, Sarni RO, Mallozi MC, Solé D. The role of nutrition in the first year of life on the prevention of allergic diseases. Rev Bras Alerg Imunopatol [Internet]. $2009 \mathrm{Mar} / \mathrm{Apr}$ [citer 2019 Aug 10]; 32(2):68-71. Available from: http://bases.bireme.br/cgibin/wxislind.exe/iah/online/?IsisScript=iah/iah. xis\&src=google\&base $=$ LILACS\&lang=p\&nextAc tion $=$ Ink\&exprSearch $=535170$ \&indexSearch $=$ ID

17. Guilherme AQ, Carvalho BC, Diniz BP, Bianco BF, Andrade TG, Araújo LA. Approach to atopic dermatitis in children by the Family Physician. Rev Bras Med Fam Comun [Internet]. 2014 Apr/June [cited 2019 Aug 10]; 9(31):159-68. DOI: https://doi.org/10.5712/rbmfc9(31)846

18. Tintori SM, Carvalho MDB, Solé D, Odebrech L, Antoniassi PC, Costa RG. Prevallence of asthma and allergic diseases in brazilian school children aged 12 to 15 years in the city of Maringa, Parana State, Brazil. RBM Rev Bras Med [Internet]. 2013 Jan/Feb [cited 2018 Aug 1];70(1):2230. Available from: http://bases.bireme.br/cgibin/wxislind.exe/iah/online/?IsisScript=iah/iah. xis\&src=google\&base $=$ LILACS\&lang=p\&nextAc tion $=$ Ink\&exprSearch $=683441$ \&indexSearch $=$ ID

19. Leite RMS. Viabilidade de o aleitamento exclusivo prevenir o desenvolvimento da dermatite atópica em lactentes até um ano de idade [dissertation][Internet]. Brasília: Universidade de Brasília; 2006 [cited 2019 Aug 15]. Available from: https://repositorio.unb.br/handle/10482/5016

20. Lee SY, Kang MJ, Kwon JW, Park KS, Hong SJ. Breastfeeding might have protective effects on atopy in children with the CD14C-159T CT/CC Genotype. Allergy Asthma Immunol Res. 2013 Jul; 5(4):239-41. DOI: http://dx.doi.org/10.4168/aair.2013.5.4.239

21. Barnes PJ. Doenças do Sistema Respiratório- Asma. In: Medicina Interna de Harrison Pneumologia: AMGH Editora; 2017. p. 1669-81.

22. Julia V, Macia L, Dombrowicz D. The impact of diet on asthma and allergic diseases. Nat Rev Immunol. 2015 May; 15(5):308-22. DOI:http://dx.doi.org/10.1038/nri3830

23. Flohr C, Mann J. New insights into to the epidemiology of childhood atopic dermatites. Allergy. 2014 Jan; 63:3-16. DOI: http://dx.doi.org/10.1111/all.12270 
24. Tezza G, Cattazzo E, Boner AL. The house of the allergic child. Pneumologia Pediatrica [Internet]. 2011 [cited 2019 Aug 10];43:5-14. Available from: http://www.docvadis.it/napolitanopediatrics/document/napolitanopediatrics/la_casa_del_bam bino_allergico/fr/metadata/files/0/file/LA\%20CASA\%20DEL\%20BAMBINO\%20ALLERGICO $\% 2$ 0-\%20PUBBLIC.pdf

25. Godinho R, Lanza M, Godinho A, Rodriques A, Assiz TML. Frequency of positive skin tests for airborne allergic agents. Rev Bras Otorrinolaringol. 2003 Nov/Dec; 69(6):824-28. DOI: http://dx.doi.org/10.1590/S0034-72992003000600016

26. Castro APM, Solé D, Rosário Filho NA, Jacob CMA, Rizzo MCFV, Fernandes MFM, et al. Practical guide for management of atopic dermatitis - conjunct opinion of allergologists from the Associação Brasileira de Alergia e Imunopatologia and Sociedade Brasileira de Pediatria. Rev Bras Alerg Imunopatol. $2006 \mathrm{Dec}$ [cited 2019 Aug 10];29(6):268-82. Available from: http://aaai-asbai.org.br/detalhe_artigo.asp?id=278

27. Melo RMB, Lima LS, Sarinho ESC. Relationship between home environmental control and exacerbation of asthma in children and adolescents in the city of Camaragibe in the state of Pernambuco, Brazil. J Bras Pneumol. 2005 Jan/Fev;31(1):5-12. DOI: http://dx.doi.org/10.1590/S1806-37132005000100003

28. Hilzendeger C, Tabalipa IO, Vanhoni LR, Oliveira SM, Piovezan AP, Silva J. Prevalence of atopic dermatitis in school adolescents in São José-SC. Arq Catarin Med [Internet]. 2010 [cited 2019 Aug 10];39(1):45-9. Available from: https://www.researchgate.net/profile/Clarissa_Hilzendeger/publication/256195421_Prevalenc e_of_atopic_dermatitis_in_school_adolescents_in_Sao_Jose_-

__C_/links/0deec521f2ff9a52ed000000/Prevalence-of-atopic-dermatitis-in-school-adolescentsin-Sao-Jose-SC.pdf

29. Carnauba $L A B$, Nunes $C P$. The impact on the quality of life of individuals with atopic dermatites. Rev Med Fam Saúde Mental [Internet]. 2019 [cited 2019 Aug 10]; 1(1): 95-108. Available from:

http://www.revista.unifeso.edu.br/index.php/medicinafamiliasaudemental/article/download/1 $567 / 619$

30. Távora EMO, Cunha MS, Leite MB, Borges BEC, Távora RCO, Azevedo VD. Evidence of the relationship between atopical dermatitis and the development of the atopical march: integrative review. Braz J Heal Rev [Internet]. 2019 Jul/Aug [cited 2019 Aug 10]; 2(4):3613-33. DOI: http://dx.doi.org/10.34119/bjhrv2n4-125

31. Medeiros ML, Solé D, Costa ADP, Andrade ANV, Mello PKS, Santos DAM, et al. Prevalence of asthma and rhinitis among adolescents aged 13-14 years in a Brazilian northeastern state capital according to the International Study of Asthma and Allergies in Childhood (ISAAC) questionnaire. Braz J Allergy Immunol. 2014; 2(3):112-8. Available from: http://aaaiasbai.org.br/audiencia_pdf.asp?aid2=694\&nomeArquivo=v2n2a05.pdf\&ano=2014. 